\title{
Primary spontaneous coronary dissectlon in a young male and the role of intravascular ultrasonography for diagnosis and treatment
}

\author{
Sadık Volkan Emren a,*, Oktay Şenöz ${ }^{b}$, Hamza Duygu $^{c}$, Cem Nazlı ${ }^{\mathrm{d}}$, Oktay Ergene ${ }^{\mathrm{e}}$ \\ a Department of Cardiology, Afyonkarahisar State Hospital, Afyonkarahisar, Turkey \\ b Department of Cardiology, Artvin State Hospital, Artvin, Turkey \\ c Department of Cardiology, Near East University School of Medicine, Nicosia, Cyprus \\ d Department of Cardiology, Katip Celebi University Atatürk Training and Research Hospital, Izmir, Turkey \\ e Department of Cardiology, Dokuz Eylül University School of Medicine, Izmir, Turkey
}

\section{A R T I C L E I N F O}

\section{Article history:}

Received 5 August 2015

Received in revised form 26 October 2015

Accepted 27 October 2015

Available online 6 November 2015

\section{Keywords:}

Spontaneous coronary dissection

Acute coronary syndrome

Intravscular ultrasound

\begin{abstract}
A B S T R A C T
Primary spontaneous coronary artery dissection (SCAD) is a rare cause of acute coronary syndrome and is observed especially during gestation or the postpartum period of young healthy female patients. SCAD is much more rarely seen in young male patients. A 32 year-old male was admitted to our clinic with a typical anginal complaint that had begun after a verbal discussion in a family environment. The patient was hospitalized with a diagnosis of acute myocardial infarction without ST elevation. During coronary angiography, a lesion seen as a dissection in the proximal region of the left anterior descending artery (LAD), 30\% stenosis in the circumflex artery and plaque in the right coronary artery were observed. In the LAD osteal region, a dissection flap and intimal rupture was observed at the 12:00-2:00 o'clock position using intravascular ultrasonography (IVUS). Our case was very young and had no atherosclerotic risk factors except for a history of smoking. It was suggested that intense emotional stress was the triggering factor for coronary dissection. The gold standard imaging method for spontaneous coronary dissection is coronary angiography. Recently, imaging methods like IVUS have made important contributions to the diagnosis of dissections that cannot be detected by coronary angiography. Treatment must be individual since there is no standard protocol. Medical therapy, percutaneous coronary intervention and coronary artery bypass surgery are the main treatment options.

(C) 2015 The Society of Cardiovascular Academy. Production and hosting by Elsevier B.V. All rights reserved.

This is an open access article under the CC BY-NC-ND license (http://creativecommons.org/licenses/by-nc-nd/4.0/).
\end{abstract}

\section{Introduction}

Primary spontaneous coronary artery dissection (SCAD) is a rare cause of acute coronary syndrome and sudden cardiac death. ${ }^{1}$ It is observed especially during gestation or the postpartum period of young healthy female patients who do not have classical cardiovascular risk factors. ${ }^{2}$ SCAD is much more rarely seen in young male patients. Early diagnosis is highly important to be able to start available treatment procedures. $^{3}$ Intravascular ultrasonography (IVUS) is a helpful imaging method that provides early diagnosis and appropriate stent size implantation.

In this paper, a case of a 32-year-old is presented, who had a stent implantation after emotional stress acute coronary syndrome because of left descending artery dissection detected by coronary angiography and IVUS.

\footnotetext{
* Corresponding author. Tel.: +90 5052644578; fax: +90272 2147575.

E-mail addresses: vemren@hotmail.com (S.V.Emren), oktaysenoz@hotmail.com

(O. Şenöz), hamzakard@yahoo.com (H. Duygu), cemekomed@hotmail.com (C. Nazlı), oktayergene@yahoo.com (O. Ergene).

Peer review under responsibility of The Society of Cardiovascular Academy.
}

\section{Case presentation}

A 32-year-old male was admitted to our clinic with a typical anginal complaint, which had begun after a verbal disscussion in a family environment. His history included smoking 10 packs of cigarettes per year. There was no disease or medication use in his history. There was also no significant condition in his background.

A sinus rhythm was detected in electrocardiography with an ST segment depression at V1-V6 derivations. HemoglobineThe initially evaluated Troponin I level was $5.3 \mathrm{ng} / \mathrm{ml}$ (reference value: $0-0.6 \mathrm{ng} / \mathrm{ml}$ ). An ejection fraction of $50 \%$ with mild aorta insufficiency and mild hypokinesia of the anterior wall were detected in echocardiography, while all other valvular structures were normal.

The patient was hospitalized with a diagnosis of acute myocardial infarction without ST elevation and coronary angiography (CAG) was planned. During coronary angiography, a lesion seen as a dissection in the proximal region of the left anterior descending artery (LAD), 30\% stenosis in the circumflex artery and plaque in the right coronary artery were observed. The dissection imaged lesion in the LAD was passed with a $0.014 \mathrm{~mm}$ guidewire and imaging with an Atlantis SR Pro $40 \mathrm{MHz}$ (Boston Scientific, Freemont, CA) intravascular ultrasonography 


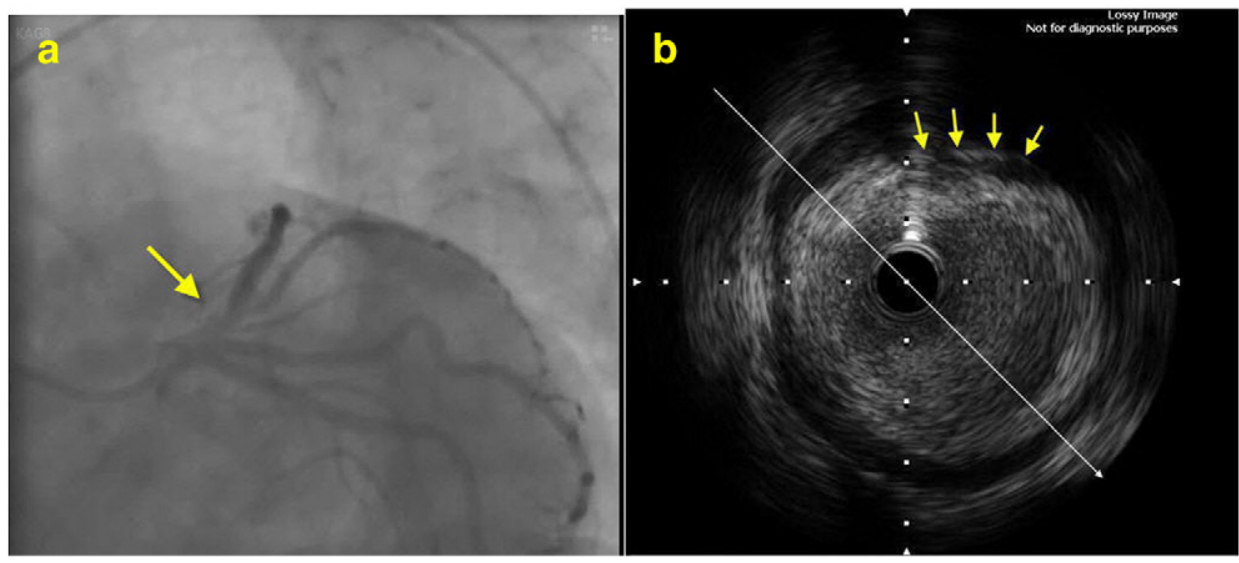

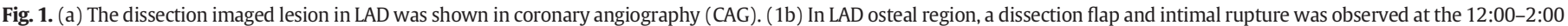
o'clock position by imaging via intrvascular ultrasonography (IVUS).

(IVUS) catheter. In the LAD osteal region, a dissection flap and intimal rupture were observed at the 12:00-2:00 o'clock position (Fig. 1). The maximum LAD diameter was $4.8 \mathrm{~mm}$ in the osteal region as measured by IVUS. A stent of $5.0^{*} 12 \mathrm{~mm}$ in size was implanted by inflating at 12 atmospheres pressure to the dissected lesion in the LAD osteal region. Imaging by IVUS after the procedure showed that the stent apposition was not appropriate. For this reason, the balloon was again put through the stent and inflated at 16 atmospheres pressure. Control imaging by IVUS showed an exactly appropriate apposition of the stent structure (Fig. 2). The patient was discharged with a prescription of acetylsalicylic acid, clopidogrel and atorvastatin.

\section{Discussion}

Spontaneous coronary artery dissection is defined as a rupture in the coronary artery wall caused by traumatic and non-iatrogenic reasons. This process results in myocardial ischemia caused by disruption of blood flow in the real lumen because of pseudo lumen formation and intramural hematoma.

Many etiologic conditions causing non-atherosclerotic SCAD exist. These are mainly fibromuscular dysplasia, gestation, connective tissue disorders, systemic inflammatory dieases, hormonal therapy and idiopathic causes. The reasons triggering SCAD include intense exercise, intensive emotional stress, birth labor, conditions with powerful valsalva maneuver such as heavy coughing, vomiting, and drugs such as cocaine and amphetamine. ${ }^{4}$ Spontaneous coronary artery dissection is more common in the female population. This fact makes us consider that hormonal factors may play an important role. It is well known that hormonal factors have a role in medial degeneration of collagen synthesis. No descriptive data exist for male cases. Our case was very young and had no atherosclerotic risk factors except a history of smoking. It was suggested that intense emotional stress was the triggering factor for coronary dissection.

The number of case reports presenting post-emotional stress coronary artery dissection is very low. Firstly, in 1998, Pamar et al. detected coronary artery dissection by coronary angiography in 2 of 4 patients with no coronary artery disease, but who experienced horrible nightmares. ${ }^{5}$ Hendir et al. reported only one emotional stress-related SCAD case in 1100 acute myocardial infarction patients. ${ }^{6}$ Mayr et al. have described a 51-year-old woman who had SCAD at work after intense emotional stress. ${ }^{7}$ Kaya et al. described acute inferior myocardial infarction due to spontaneous coronary artery dissection in a 21-yearold medical school student who experienced examination stress. ${ }^{8}$ The fact that our case experienced severe angina pectoris after an intense argument in the family made us consider that emotional stress might be a triggering factor in development of the spontaneous coronary dissection.

Coronary angiography is the most commonly used imaging method in the diagnosis of SCAD. ${ }^{9}$ SCAD can be diagnosed during CAG by demonstration of a radiolucent intimal flap frequently associated with contrast staining on the vessel wall. Although CAG can clearly show the lumen of the vessel wall, it cannot depict the vessel wall. Therefore, it is difficult to diagnose SCAD with development of intramural hematoma. Recently, IVUS has been able to provide novel diagnostic insights in patients with SCAD. Coronary lumen and vessel walls are nicely depicted with this technque. IVUS can demonstrate clearly dissected membrane, and true and false lumen, which cannot be detected on coronary angiography in patients with silent SCAD. ${ }^{10}$ IVUS can provide clear pictures of intramural hematoma and false lumen thrombosis,

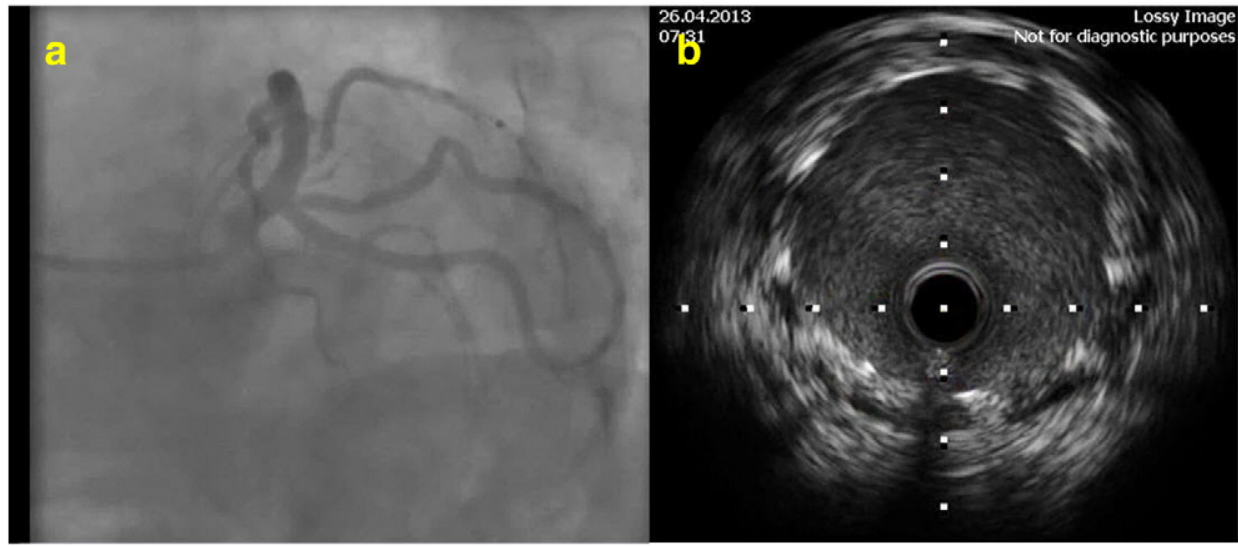

Fig. 2. (a) Dissected lesion in LAD was closed after stent implantation. (2b) IVUS image showed the apposition of stent structures. 
which can be misdiagnoesd as atherosclerotic narrowing on CAG. Although we could see a lesion like an intimal flap in the proximal region of the LAD during CAG, SCAD was confirmed clearly with IVUS by demonstrating a dissected flap, and also the exact localization of the lesion could be depicted.

IVUS has also been used to optimize the results of stent implantation in patients with SCAD requiring coronary intervention. Stent size and length may be selected by considering the true vessel size and the true longitudinal extension of the dissection, thereby ensuring optimal stent appositon to the vessel wall. ${ }^{11}$ In our case, we determined the stent size according to the IVUS measurement. Indeed, insufficent stent expansion and apposition could be depicted after reevaluating with IVUS. Therefore, it was necessary to postdilate with a balloon catheter. After postdilatation, optimal stent expansion, stent appositon and complete closure of the dissection line can be ensured with IVUS guidance.

Treatments must be individual because there is no standard protocol. Medical therapy, percutaneous coronary intervention and coronary artery bypass surgery are the main treatment options. ${ }^{12}$ Percutaneous coronary intervention was preferred in our case because of the continuous anginal complaint, LAD osteal region dissection development and wide ischemia of the myocardium. We continued dual antiplatelet therapy for 1 year after stent implantation. Prophylactic statin therapy was commenced due to detection of non-critical coronary stenosis in other vessels

\section{Conclusion}

Although spontaneous coronary dissection is a rare cause of acute coronary syndrome and sudden cardiac death, it must be considered and investigated in cases with low atherosclerotic risk factors but typical anginal complaints, and IVUS imaging must be applied if necessary.

\section{References}

1. Basso C, Morgagni GL, Thiene G. Spontaneous coronary artery dissection: a neglected cause of acute myocardial ischaemia and sudden death. Heart 1996;75:451.

2. Giacoppo D, Capodanno D, Dangas G, et al. Spontaneous coronary artery dissection. Int J Cardiol 2014;175:8-20.

3. Saw J. Spontaneous coronary artery dissection. Can J Cardiol 2013;29:1027-1033.

4. Yip A, Saw J. Spontaneous coronary artery dissection-A review. Cardiovasc Diagn Ther 2015;5(1):37-48.

5. Parmar MS, Luque-Coqui AF. Killer dreams. Can J Cardiol 1998;14:1389-1391.

6. Hendiri T, Bonvini RF, Martin W, Doriot PA, Camenzind E. Acute myocardial infarction due to spontaneous coronary artery dissection. Arch Mal Coeur Vaiss 2005;98: 974-978.

7. Mayr A, Klug G, Jaschke W, Pachinger O, Metzler B. Persistent spontaneous dissection of the left anterior descending coronary artery after emotional pressure. Wien Klin Wochenschr 2010;122:515-517.

8. Kaya Y, Çağlıyan ÇE, Ceyla Y, Balcı B. Acute myocardial infarction due to coronary artery dissection triggered by emotional stress Kafkas. J Med Sci 2012;2(3):125-127.

9. Alfonso F, Bastante T, Rivero F, et al. Spontaneous coronary artery dissection. Circ J 2014;78:2099-2110.

10. Alfonso F, Bastante T, Cuesta J, Rodríguez D, Benedicto A, Rivero F. Spontaneous coronary artery dissection: novel insights on diagnosis and management. Cardiovasc Diagn Ther 2015;5(2):133-140.

11. Maehara A, Mintz GS, Castagna MT, et al. Intravascular ultrasound assessment of spontaneous coronary artery dissection. Am J Cardiol 2002;89:466-468.

12. Capuano C, Sesana M, Predolini S, Leonzi O, Cuccia C. Case report: a very large dissection in the left anterior descending coronary artery of a 56-year-old man. Cardiovasc Revasc Med 2006;7:240-242. 